\title{
TOPOLOGICAL SPACES AND LAT TICES OF LOWER SEMICONTINUOUS FUNCTIONS
}

BY

\author{
M. C. THORNTON
}

ABSTRACT. Lower semicontinuous real-valued functions on a space $X$ form a conditionally complete distributive lattice $L(X)$. Those lattices which can be represented as $L(X)$ for some $X$ are characterized algebraically. All spaces producing isomorphic lattices are determined. The class of spaces which are determined by their function lattices is introduced.

1. Introduction and notation. The purpose of this work is to examine the relations between the topology of a space $X$ and the lattice of its lower semicontinuous real-valued functions $L(X)$. The following questions are answered: Which abstract lattices can be represented as $L(X)$ for some space $X$ ? Which spaces give isomorphic lattices? What are necessary and sufficient conditions on a space $X$ to have the property that for $T_{0}$ spaces $Y, L(X)$ isomorphic to $L(Y)$ implies $X$ is homeomorphic to $Y$ ? When can the topology be reconstructed from the lattice? Up to some homeomorphisms, the collection of spaces with is omorphic lattices can be considered as a lattice $S$ under inclusion. $S$ always has a maximum element $X_{1}$ and sometimes a minimum element $X_{0}$. If two spaces $X_{s}$ and $X_{t}$ of $S$ are homeomorphic there is a lattice automorphism of $S$ taking $X_{s}$ to $X_{t}$.

The above questions were first considered and very nicely answered for the lattice $C(X)$ of closed subsets of a space by Drake and Thron [4] and later by Blanksma [3]. Both papers also considered the corresponding lattice $S$. Using recent work of $\mathrm{Nel}$ and $\mathrm{W}$ ilson $([5],[6])$, the general approach for the lattices $C(X)$ can be modified to apply to the lattices $L(X)$. The details however are much more complicated. Instead of single elements, equivalence classes of ideals of elements must be considered. The relationship between the topologies and the lattices is best expressed in terms of functors between the topological category and a suitable lattice category. For this reason, the material here is organized along the lines of [3].

Received by the editors April 14, 1972 and, in revised form, October 20, 1972.

AMS (MOS) subject classifications (1970). Primary 54C30, 54C40; Secondary 06A35, 26A 15.

Key words and phrases. Lower semicontinuous functions, lattice of closed subsets, fc spaces. 
Since the characteristic functions for the open subsets are lower semicontinuous, the lattice $L(X)$ contains at least as much information about the topology as $C(X)$. To show the lattice $L(X)$ may say more about the topology, consider the space $Y=[0,2]$ with $\{(r, 2] \mid r \in[0,2]\}$ as a basis for the open sets. Let $Y_{t}=Y-t$ with the induced topology. The spaces $Y, Y_{0}, Y_{1}$, and $Y_{2}$ are topologically distinct. The $C$ lattices distinguish between $Y$ and $Y_{0}$ but not between $Y$ and $Y_{2}$. The $L$ lattices distinguish between $Y$ and $Y_{0}$, and between $Y$ and $Y_{2}$ but not between $Y$ and $Y_{1}$.

A lower semicontinuous real-valued function $f$ of $X$ will be viewed as a map $f: X \rightarrow R$ where here and following $R$ is the reals $w$ ith the topology generated by $\{(t, \infty) \mid t \in R\}$. The lattice $L(X)$ has as its elements all such maps. In $L(X), f<g$ iff $f(x) \leq g(x)$ for each $x \in X$. Then $f \vee g=\max (f, g)$ and $f \wedge g=$ $\min (f, g)$. With these definitions, $L(X)$ is known to be a conditionally complete distributive lattice. A subset $I$ of a lattice $M$ is an ideal if $f, g \in I$ implies $f \vee g \in I$ and $f \in I, g \in M$ implies $f \wedge g \in I . I$ is a prime ideal if $f \wedge g \in I$ implies either $f$ or $g$ is in $I$. $I$ is called closed if whenever $f_{i} \in I$ and $\bigvee f_{i}$ exists, then $\bigvee f_{i}$ is also in $I$.

All spaces considered will be $T_{0}$ spaces. The usual closure operator will be written $c l$. Derived sets will be denoted by a prime. In particular $x^{\prime}$ is the derived set of a point. The front closure operator, $\mathrm{fcl}$, is defined by $x \in \mathrm{fcl} A$ iff $x \in \operatorname{cl}(\operatorname{cl} x \cap A)$. If $\mathrm{fcl} A=X$, then $A$ is fcl-dense in $X$. The fcl closure operator is the same as the $b$-closure of [7] and determines equalizers for $T_{0}$ spaces [6, Theorem 2.1]. A subset $A \subset X$ has the filter countable intersection property or FCI-property, if every open filter $\left\{G_{\alpha}\right\}$ in $X$ with $G_{\alpha} \cap A \neq \varnothing$ for each $\alpha$ has nonempty countable intersections. Lemma 4.1 of [6] gives an equivalent condition: $A \subset X$ has the FCI-property iff every map $f: X \rightarrow R$ is bounded above on $A$. A subset is irreducible closed if it is not the proper union of two closed sets. Note that $\mathrm{cl} x$ is an irreducible closed set with the FCI-property since any $f$ has an upper bound of $f(x)$ on $\mathrm{cl} x$. A space with the property that an irreducible closed set is a point closure is called a pc space. If irreducible closed sets with the FCI-property are point closures, the space is called an $\mathrm{fc}$ space. FCI, pc, and fc were defined and studied in [6].

2. The functors $L$ and $T$. To discover a characterization of those lattices $M$ which can be represented as $L(X)$ for some $X$, we first consider some necessary conditions. Suppose $M=L(X)$. For each real number $r$, the translation $t_{r}: R \rightarrow R$ given by $t_{r}(s)=r+s$ is a map. Define a left $R$-action on $M, \Gamma: R$ $\times M \rightarrow M$ by $\Gamma(r, f)=t_{r} f$. Then $\Gamma_{r}=\Gamma \mid r \times M$ is a lattice automorphism $M \rightarrow M$ such that $\Gamma_{0}(f)=f, \Gamma_{r}(f)>f$ if $r>0$, and $\Gamma_{r}(f)<f$ if $r<0$. Note $\Gamma_{r}$ is fixed point free if $r \neq 0$. Two ideals $I$ and $J$ will be called equivalent if there is a $\Gamma_{r}$ such that $\Gamma_{r}(I)=J$. 
Let $A$ be a closed subset of $X$. Then $l(s, A)=\{f: X \rightarrow R \mid f(a) \leq s$ for all $a \in A\}$ is an ideal of $M$. By [5, Proposition 3], if $A$ is irreducible closed, then $I(s, A)$ is a closed prime ideal and moreover, every closed prime ideal of $M$ is of this form. Since $\Gamma_{r}(I(s, A))=I(r+s, A), I(A)=\{I(s, A) \mid s \in R\}$ is a complete equivalence class of closed prime ideals. Let $\Lambda$ be the set of all irreducible closed subsets of $X$ with the FCI-property. As noted before, cl $x \in \Lambda$ for each $x$ in $X$. We shall be concerned with the collection of equivalence classes $\{I(A) \mid A \in \Lambda\}$. The closed prime ideal $I(0, A)$ will be considered as the standard representative of the class $I(A)$.

Given any $f \in M$ and $I(A)$ with $A \in \Lambda$ there is a minimum number $r$ such that $f \in I(r, A)$. In fact $r=\sup \{f(x) \mid x \in A\}$. If $A=\mathrm{cl} x$, then $r=f(x)$. For this reason, let minimum $r$ corresponding to $A$ be denoted by $f(A)$. It is easily seen that $f=\bigvee \bigcap\{I(f(x), \operatorname{cl} x) \mid x \in X\}$ and so also $f=\bigvee \bigcap\{I(f(A), A) \mid A \in \Lambda\}$. A subcollection $\Sigma=\{I(B)\}$ of classes is called hull-kernel closed if $I(0, C) \supset$ $\cap I\left(0, B_{i}\right)$ and $I\left(B_{i}\right) \in \Sigma$ implies $I(C) \in \Sigma$. Let $\left\{I\left(s_{A}, A\right) \mid A \in \Lambda\right\}$ be a set of representatives with the property that for each $s$, the set $\Sigma_{s}=\left\{I(A) \mid s_{A} \leq s\right\}$ is hull-kernel closed. Define the function $f: X \rightarrow R$ by $f(x)=s_{\mathrm{cl} x}$. To show $f$ is continuous, consider $f^{-1}((-\infty, s])=\left\{x \mid s_{\mathrm{cl} x} \leq s\right\}$. If $y \in \operatorname{cl}\left\{x \mid s_{\mathrm{cl} x} \leq s\right\}$, then $\operatorname{cl} y \subset \mathrm{cl} \cup\left\{\mathrm{cl} x \mid s_{\mathrm{cl} x} \leq s\right\}$. This implies $l(0, \mathrm{cl} y) \supset \bigcap l(0, \mathrm{cl} x)$ where $s_{\mathrm{cl} x}$ $\leq s$. Since $I(\operatorname{cl} x) \in \Sigma_{s}$ which is assumed hull-kernel closed, $I(\operatorname{cl} y) \in \Sigma_{s}$ or $s_{\mathrm{cl} y} \leq s$. Therefore $y \in\left\{x \mid s_{\mathrm{cl} x} \leq s\right\}$ and $f$ is continuous. By the way $f$ was defined, $f=\bigvee \bigcap I\left(s_{A}, A\right)$. Also $f(A)=s_{A}$ can be verified.

The existence of a $\Gamma$-action and the closed prime ideals $I(s, A)$ turns out to be a sufficient condition we were seeking. Explicitly, define an allowable $R$-action on a lattice $M$ to be a function $\Gamma: R \times M \rightarrow M$ such that $\Gamma_{r}$ given by $\Gamma_{r}(f)=\Gamma(r, f)$ is an automorphism, $\Gamma_{0}(f)=f, \Gamma_{r}(f)>f$ if $r>0, \Gamma_{r}(f)<f$ if $r<$ 0 , and $\Gamma_{r} \Gamma_{s}=\Gamma_{r+s}$. Two closed prime ideals of $M$ will be called $\Gamma$-equivalent if there is a $\Gamma_{r}$ taking one onto the other. We write these equivalence classes as $P(A)=\{P(r, A) \mid r \in R\}$ where $A \in \Lambda$ is viewed as an index for the classes. The members of each class will be indexed by the reals so that $\Gamma_{r}(P(s, A))=$ $P(r+s, A)$. An equivalence class $P(A)$ is called bounded if for any $f \in M$ there is a minimum number $t$ such that $f \in P(t, A)$. As before we write $f(A)$ for this minimum $t$. A collection $\Sigma=\{P(A)\}$ is called hull-kernel closed if $P(0, B) \supset$ $\bigcap P\left(0, C_{i}\right) \neq \varnothing$ and $\left\{P\left(C_{i}\right)\right\} \subset \Sigma$ implies $P(B) \in \Sigma$. An $R$-basis of closed prime ideals is a collection $W=\{P(A) \mid A \in \Lambda\}$ of bounded equivalence classes with two properties: (i) $f=\bigvee \bigcap\{P(f(A), A) \mid P(A) \in W\}$ and (ii) If $\left\{P\left(s_{\alpha}, A_{\alpha}\right) \mid\right.$ $\left.P\left(A_{\alpha}\right) \in W\right\}$ is a set of representatives such that $\Sigma_{r}=\left\{P\left(A_{\alpha}\right) \mid s_{\alpha} \leq r\right\}$ is hullkernel closed for every real $r$, then the expression $\bigvee \bigcap\left\{P\left(s_{a}, A_{\alpha}\right) \mid P\left(A_{\alpha}\right) \in W\right\}$ defines a function $f$ which satisfies $f\left(A_{\alpha}\right)=s_{\alpha}$. A lattice has sufficient closed prime ideals if the collection of all bounded classes of closed prime ideals forms an $R$-basis. 
Theorem 1. A lattice $M$ is isomorphic to $L(X)$ for some topological space $X$ iff $M$ is a conditionally complete distributive lattice which bas an allowable $R$-action and an R-basis of closed prime ideals.

Proof. By the previous remarks if $M=L(X)$ then the translations define the allowable $R$-action and $\{I(A)\}$ where $A$ is an irreducible closed set with the FCI-property gives an $R$-basis of closed prime ideals. Conversely suppose the lattice $M$ with action $\Gamma$ and $R$-basis $W=\{P(A)\}$ is given. Define the topological space $X=\Theta(M, W)$ by saying the points of $X$ are the elements of $W$ and $\Sigma \subset X$ is closed iff $\Sigma$ is hull-kernel closed in $W$. Arbitrary intersections of closed sets, $\varnothing$, and $X$ are easily seen to be closed. Suppose $\Sigma_{1}$ and $\Sigma_{2}$ are two closed sets and $P(0, C) \supset \bigcap P(0, B) \cap \bigcap P(0, D)$ where $P(B) \in \Sigma_{1}$ and $P(D) \in \Sigma_{2}$. If $P(0, C) \not \supset \bigcap P(0, B)$ and $P(0, C) \not \supset \bigcap P(0, D)$ choose. $f \epsilon$ $\bigcap P(0, B)-P(0, C)$ and $g \in \bigcap P(0, D)-P(0, C)$. Then $f \wedge g \in \bigcap P(0, B) \cap$ $\bigcap P(0, D)$ so $f \wedge g \in P(0, C)$. This contradicts the primeness of $P(0, C)$. Therefore, say, $P(0, C) \supset \bigcap P(0, B)$ and $P(C) \in \Sigma_{1} \cup \Sigma_{2}$. Thus finite unions of closed sets are closed and $X$ is a topological space. With this topology, the closure of $P(A)$ is easily seen to be $\{P(C) \mid P(C) \in W, P(0, C) \supset P(0, A)\}$. Thus if $\mathrm{cl} P(A)=\mathrm{cl} P(B), P(0, A) \supset P(0, B)$ and $P(0, B) \supset P(0, A)$ or $P(A)=P(B)$.

Therefore $X$ is a $T_{0}$ space.

For each $f \in M$ define the function $\theta f: X \rightarrow R$ by $\theta f(P(A))=f(A)$. To show $\theta f$ is continuous, consider $\theta f^{-1}((-\infty, r])=\{P(A) \mid f(A) \leq r\}=\{P(A) \mid f \in P(r, A)\}$ and suppose $P(0, C) \supset \bigcap P(0, A)$ where $f \in P(r, A)$. Then $f \in \bigcap P(r, A) \subset$ $P(r, C)$ so $P(C) \in \theta f^{-1}((-\infty, r])$ which is therefore closed. Note that if $f<g$ as elements of $M$, then $g \in P(t, A)$ implies $f \in P(t, A)$ or $f(A) \leq g(A)$. Thus $\theta f \leq \theta g$ so $\theta: M \rightarrow L(X)$ is a lattice homomorphism. Now suppose $b: X \rightarrow R$ is continuous. Then $b^{-1}((-\infty, r])=\{P(A) \mid b(P(A)) \leq r\}$ is closed for each $r$. Consider the collection of representatives $\{P(b(P(A)), A)\}$. According to condition (ii) of the base $W, \bigvee \cap P(b(P(A)), A)$ exists and we call it $\psi h$. Then $\psi h(A)=b(P(A))$. This defines a function $\psi: L(X) \rightarrow M$ given by $b \rightarrow \psi h$. If $b \leq k$ in $L(X)$ then $P(b(P(A)), A) \subset P(k(P(A)), A)$ for all $P(A) \in X$. Thus $\psi b$ $\leq \bar{\psi} k$ and $\psi$ is a lattice homomorphism. $\psi \theta f=\bigvee \cap P(f(A), A)$ which by condition (i) of $W$ is $f$. $\theta \psi b$ is easily seen to be $b$. Thus $\theta$ and $\psi$ are inverses and $\theta: M \rightarrow L(X)$ is a lattice isomorphism.

The characterization of lattices representable as lower semicontinuous functions given in Theorem 1 depends upon the additive structure of the reals. A characterization solely in lattice terms would be more satisfying. Analogous results of Anderson and Blair [1] which characterize rings representable as rings of continuous functions use the notions of pseudoregular, maximal real ideals, $R$-extension, and $S$-spectra which involve the reals in an essential manner. Char- 
acterizing lattices representable as lower semicontinuous functions or rings representable as continuous functions without use of the reals appears to be very difficult or perhaps impossible.

Conditionally complete distributive lattices which have allowable $R$-actions and sufficient closed prime ideals will be the objects in the category called Lat. The morphisms in Lat are to arise from and produce continuous functions in Top, the category of $T_{0}$ spaces and maps. The following conditions on lattice homomorphisms are exactly those necessary in order to achieve this. Let $M$ and $N$ be objects in Lat with $R$-actions $\Gamma, \Gamma^{\prime}$ and bounded closed prime ideal classes $W=\{P(A)\}, W^{\prime}=\left\{P^{\prime}(B)\right\}$ respectively. A function $\phi: N \rightarrow M$ is a morphism in Lat iff $\phi$ satisfies

(1) $\phi\left(\bigvee_{\alpha} a_{\alpha}\right)=\bigvee_{\alpha} \phi\left(a_{\alpha}\right)$;

(2) $\phi(a \wedge b)=\phi(a) \wedge \phi(b)$;

(3) $\phi\left(\Gamma_{r}^{\prime}(a)\right)=\Gamma_{r}(\phi(a)), r \in R$;

(4) for each $P(A) \in W$, there is a $P^{\prime}(B) \in W^{\prime}$ with $\phi\left(P^{\prime}(s, B)\right) \subset P(s, A)$ and such that $\phi(b) \in P(s, A)$ implies $b \in P^{\prime}(s, B)$.

Note that the class $P^{\prime}(B)$ in (4) is unique and because of $(3), \phi(b) \in P(t, A)$ implies $b \in P^{\prime}(t, B)$ for all $t$.

Theorem 2. For $X \in T o p$, let $L(X)$ be the lattice of maps $X \rightarrow R$ with an $R$-action given by translations of $R$. For $f: X \rightarrow Y$ define $L(f): L(Y) \rightarrow L(X)$ by $L(f)(g)(x)=g(f(x))$. Then $L:$ Top $\rightarrow$ Lat is a contravariant functor.

Proof. Let $L(X)=M, L(Y)=N$ and $L(f)=\phi . L$ is easily seen to preserve compositions and identities. $\phi$ clearly satisfies properties (1), (2), and (3). To verify (4), let $I(s, A) \subset M$ with $A$ an irreducible closed FCI set. Then cl $f(A)$ $=B$ is a closed set which is also seen to be irreducible and FCI in $Y$. Thus $I(B)$ is a bounded class of closed prime ideals in $N$. Let $g \in I(s, B)$ and $x \in$ $A$. Then $\phi(g)(x)=g(f(x)) \leq s$ since $f(x) \in B$. Therefore $\phi(I(s, B)) \subset I(s, A)$. Suppose $g \in N$ and $\phi(g) \in I(s, A)$. Since $g(c l f(A)) \subset \mathrm{cl} g(f(A)) \subset(-\infty, s]$, we have $g \in I(s, B)$ as required.

Theorem 3. For $M \in$ Lat, let $T(M)$ be the space whose points are the bounded classes of closed prime ideals in $M$ and whose closed sets are the bull-kernel closed subsets. For $\phi: N \rightarrow M$ define $T(\phi): T(M) \rightarrow T(N)$ by $T(\phi)(P(A))=P^{\prime}(B)$ where $P^{\prime}(B)$ is determined by property (4). Then $T:$ Lat $\rightarrow$ Top is a contravariant functor.

Proof. It is only necessary to prove that $T(\phi): T(M) \rightarrow T(N)$ is continuous. Let $\Sigma=\left\{P^{\prime}(B)\right\}$ be a closed set of $T(N)$. To.show $T(\phi)^{-1}(\Sigma)=\{P(A) \mid T(\phi)(P(A))$ 
$\epsilon \Sigma\}$ is closed suppose $P(0, D) \supset \bigcap P\left(0, E_{i}\right)$ where $P\left(E_{i}\right) \in T(\phi)^{-1}(\Sigma)$. Let $T(\phi)(P(D))=P^{\prime}(F)$ and $T(\phi)\left(P\left(E_{i}\right)\right)=P^{\prime}\left(G_{i}\right)$. Thus $\phi\left(P^{\prime}\left(0, G_{i}\right)\right) \subset P\left(0, E_{i}\right)$ and

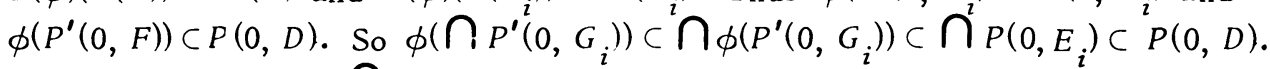
Then by property (4), $\cap P^{\prime}\left(0, G_{i}\right) \subset P^{\prime}(0, F)$. Since $P^{\prime}\left(G_{i}\right) \in \Sigma$ and $\Sigma^{i}$ is closed, $P^{\prime}(F) \in \Sigma$ or $P(D) \in T(\phi)^{-1}(\Sigma)$. Therefore $T(\phi)$ is continuous. Note that $T$ applied to a lattice $L(X)$ is the $\Omega$ construction of $[5]$ if all irreducible closed sets have the FCI-property.

To describe the relations between the functors $L$ and $T$, two functions are necessary. For $X \in T o p$, define $\tau_{X}: X \rightarrow T L(X)$ by $\tau_{X}(x)=I(\operatorname{cl} x)$. For $M \epsilon$ Lat, define $\theta_{M}: M \rightarrow L T(M)$ by $\theta_{M}(f)(P(A))=f(A)$ as in Theorem 1 . The proof of the following lemma is straightforward and is omitted. Lemma 4 and Theorem 5 are the analogues of $\$ 2$ in [3].

Lemma 4. (1) $\tau_{X}: X \rightarrow T L(X)$ is a bomeomorphism into.

(2) $\tau=\left\{\tau_{X}\right\}$ : Identity $\rightarrow T L$ is a natural transformation.

(3) $\theta_{M}: M \rightarrow L T(M)$ is a lattice isomorphism.

(4) $\theta=\left\{\theta_{M}\right\}:$ Identity $\rightarrow L T$ is a natural equivalence.

(5) $T\left(\theta_{M}\right) \tau_{T(M)}=1_{T(M)}$.

(6) $L\left(r_{X}\right) \theta_{L(X)}=1_{L(X)}$.

Theorem 5. $T$ and $L$ are adjoint functors on the right.

Proof. To show $\operatorname{Hom}_{T o p}(X, T(M))$ is naturally equivalent to $\operatorname{Hom}_{L a t}(M, L(X))$, let $g: X \rightarrow T(M)$ be a morphism in Top and $\phi: M \rightarrow L(X)$ a morphism in Lat. Define $\alpha(g)=L(g) \theta_{M}$ and $\beta(\phi)=T(\phi) \tau_{X}$. Then $\beta a(g)=\beta\left(L(g) \theta_{M}\right)=T\left(L(g) \theta_{M}\right) \tau_{X}$ $=T\left(\theta_{M}\right) T L(g) \tau_{X}=T\left(\theta_{M}\right) \tau_{T(M)}(g)=1_{T(M)}(g)=g$. Also $\alpha \beta(\phi)=\alpha\left(T(\phi) \tau_{X}\right)=$ $L\left(T(\phi) \tau_{X}\right) \theta_{M}=L\left(r_{X}\right) L T(\phi) \theta_{M}=L\left(\tau_{X}\right) \theta_{L(X)} \phi=1_{L(X)}(\phi)=\phi$. Therefore $\alpha$ and $\beta$ are inverse functions. To show $\beta$ is natural suppose $b: Y \rightarrow X$ is in Top and $\psi: N \rightarrow M$ is in Lat. Consider the diagram:

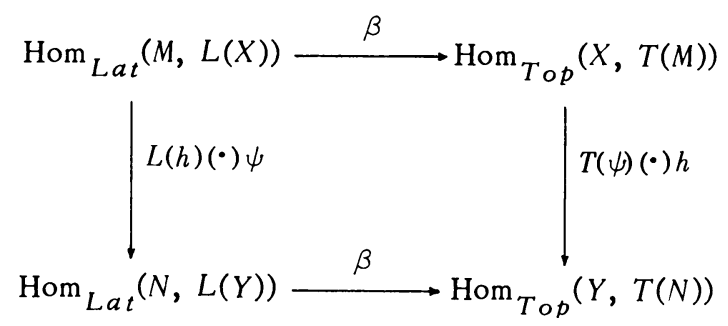

Let $\phi: M \rightarrow L(X)$. Going across then down gives $T(\psi) \beta(\phi) b=T(\psi) T(\phi) \tau_{X} b$. Going down then across yields $\beta(L(b) \phi \cdot \psi)=T(L(b) \phi \psi) \tau_{X}=T(\psi) T(\phi) T L(b) \tau_{X}=$ $T(\psi) T(\phi) \tau_{X} b$, so the diagram commutes. The naturality for $\alpha$ is proven similarly. 
3. Spaces with isomorphic lattices. Let $M \in L$ at have an $R$-basis $W$. Recall from the proof of Theorem 1 that $\Theta(M, W)$ is the space whose points are the classes in $W$ and the topology is given by the hull-kernel closed subsets. If $W$ consists of all bounded classes of closed prime ideals, then $\Theta(M, W)=T(M)$. If $W$ does not contain all bounded classes, then $\Theta(M, W) \subset T(M)$.

Theorem 6. If $L(X)$ is isomorpbic to $M$, then $X$ is bomeomorpbic to $\Theta(M, W)$ for some R-base W. In particular, $X$ is bomeomorpbic to $\Theta(L(X), W)$ where $W=$ $\{I(\operatorname{cl} x) \mid x \in X\}$.

Proof. Let $\phi: L(X) \rightarrow M$ be an isomorphism. Set $W=\{\phi I(\operatorname{cl} x) \mid x \in X\}$ where $\phi I(\mathrm{cl} x)$ is the equivalence class of $\phi(I(0, \mathrm{cl} x))$. Define a function $b$ : $X \rightarrow \Theta(M, W)$ by $b(x)=\phi I(\mathrm{cl} x)$. Clearly $b$ is one to one and onto. For a subset $C \subset X, b(C)=\{\phi I(\mathrm{cl} x) \mid x \in C\}$. Suppose $C$ is closed and $\phi I(0, \mathrm{cl} y) \supset$ $\bigcap \phi I\left(0, \mathrm{cl} x_{i}\right)$ where $x_{i} \in C$. Then $I(0, \mathrm{cl} y) \supset \bigcap I\left(0, \mathrm{cl} x_{i}\right)$ and $\mathrm{cl} y C \operatorname{cl} \cup \mathrm{cl} x_{i}$ $C C$. Thus $y \in C, \phi I(\mathrm{cl} y) \in b(C)$ and $b(C)$ is closed. Conversely, if $b(C)$ is closed and $y \in \mathrm{cl} C$, then $\mathrm{cl} y \subset \operatorname{cl} C$ so $I(0, \mathrm{cl} y) \supset \bigcap I(0, \mathrm{cl} x), x \in C$. Therefore $\phi I(0, \mathrm{cl} y) \supset \bigcap \phi I(0, \mathrm{cl} x)$ and $\phi I(\mathrm{cl} y) \in b C$. Thus $y \in C$ and $C$ is closed. Hence $b$ is a homeomorphism.

Since $L \Theta(M, W) \approx M \approx L T(M)$, the inclusion $\Theta(M, W) \subset T(M)$ gives an example of a proper subspace with the same lattice of functions. A natural question is for which subspaces $Z \subset X$ does the inclusion give an isomorphism $L(Z) \approx$ $L(X)$ ? By [5, Theorem 6], if $L(Z) \approx L(X)$ then $Z$ and $X$ have isomorphic lattices of closed sets. By $\$ \$ 2$ and 3 of [3], a necessary condition is therefore that $Z$ be superdense in $X$. Superdense was first defined by Blanksma [3] to mean property (1) of the next lemma. Here it is more convenient to view it as fcldense.

Lemma 7. Let $Z$ be a subspace of $X$. Then the following are equivalent:

(1) If $P$ and $Q$ are closed in $X$ and $P \cap Z=Q \cap Z$ then $P=Q$.

(2) If $P$ is closed in $X$, then $\mathrm{cl}(P \cap Z)=P$.

(3) $Z$ is fcl-dense in $X$.

Proof. Suppose $P$ is closed in $X$. Since cl $(P \cap Z)$ meets $Z$ in $P \cap Z$, by (1) $\mathrm{cl}(P \cap Z)=P$. Conversely if both $P$ and $Q$ are closed and if $P \cap Z=Q \cap$ $Z$ then $P=\operatorname{cl}(P \cap Z)=\mathrm{cl}(Q \cap Z)=Q$ so (1) and (2) are equivalent. Assume $Z$ satisfies (2) and let $x \in X-Z$. Then $\operatorname{cl} x=\operatorname{cl}(\operatorname{cl} x \cap Z)$ so $x \in \operatorname{cl}(\operatorname{cl} x \cap Z)=$ $\mathrm{cl}\left(x^{\prime} \cap Z\right)$ or $x \in \mathrm{fcl} Z$. Thus fcl $Z=X$. Conversely suppose fcl $Z=X$ and $P$ is closed. Clearly $\mathrm{cl}(P \cap Z) \subset P$ and $P \cap Z \subset \mathrm{cl}(P \cap Z)$. Let $p \in P-Z$. Then $U(p) \cap \operatorname{cl} p \cap Z \neq \varnothing$ and so $U(p) \cap P \cap Z \neq \varnothing$ for all open sets $U$ about $p$. 
Therefore $p \in \operatorname{cl}(P \cap Z)$ or $P-Z \subset \operatorname{cl}(P \cap Z)$. Thus $\operatorname{cl}(P \cap Z)=P$ and (3) is equivalent to (2).

Lemma 8. Let $Z$ be fcl-dense in $X$. Suppose $g: Z \rightarrow R$ is a map which is bounded above on $x^{\prime} \cap Z$ for eacb $x \in X-Z$. Then $f: X \rightarrow R$ defined by $f(x)=$ $\sup \{f(z) \mid z \in \operatorname{cl} x \cap Z\}$ is the unique continuous extension of $g$.

Proof. For $x \in Z, g^{-1}((-\infty, g(x)])$ is closed and contains $x$, so $g(z) \leq$ $g(x)$ if $z \in \operatorname{cl} x \cap Z$. Thus $f(x)=g(x)$ and $f$ extends $g$. Since equalizers are fcl-closed [6, Theorem 2.1], the extension $f$ must be unique if it exists. Since $Z$ is fcl-dense, cl $x \cap Z \neq \varnothing$ so $f(x)$ is defined for every $x$. To show $f$ is continuous, we show $f^{-1}((r, \infty))$ is open. Since $g$ is continuous, $g^{-1}((r, \infty))=$ $U$ is an open subset of $Z$. Thus there is a (unique) open $V$ in $X$ with $U=V \cap$ $Z$. Let $x \in V$. Then $V \cap \operatorname{cl} x \cap Z \neq \varnothing$ so $\operatorname{cl} x \cap U \neq \varnothing$. Thus there is a $z \epsilon$ cl $x \cap U \subset \operatorname{cl} x \cap Z$ with $g(z)>r$. Therefore $f(x)>r$ and $x \in f^{-1}((r, \infty))$. Hence $V \subset f^{-1}((r, \infty))$. Conversely, suppose $x \notin V$. Then $\operatorname{cl} x \subset X-V$ and $\operatorname{cl} x \cap Z$ $C Z-U$. Then $g \mid c l x \cap Z$ is bounded above by $r$. Thus $f(x) \leq r, x \notin f^{-1}((r, \infty))$ and $f^{-1}((r, \infty)) \subset V$. Thus $f^{-1}((r, \infty))=V$ is open.

Theorem 9. Let $i: Z \rightarrow X$ be the inclusion of an fcl-dense subspace. Then the following are equivalent:

(1) $L(i): L(X) \rightarrow L(Z)$ is an isomorphism.

(2) Let $A$ be closed in $Z$. Then $A$ bas the FCI-property in $Z$ iff $\mathrm{cl}_{X} A$ bas the FCI-property in $X$.

(3) For each $x \in X-Z, x^{\prime} \cap Z$ bas the FCI-property in $Z$.

Proof. Suppose the restriction map $L(i)$ is an isomorphism. If $\mathrm{cl}_{X} A$ has the FCI-property in $X$ and $g: Z \rightarrow R$ is given, $g$ extends to $f$ which is bounded on $\mathrm{cl}_{X} A$. Therefore $g$ is bounded on $\mathrm{cl}_{X} A \cap Z$ and $A$ has the FCI-property in $Z$. Now suppose $A$ has the FCI-property in $Z$ and $f: X \rightarrow R$ is given. Then $g=f \mid Z$ is bounded above on $A$. Since $A$ is closed in $Z, A$ is fcl-dense in $\mathrm{cl}_{X} A$. By Lemma 8, the values of $f$ on $\mathrm{cl}_{X} A$ are sups of the values of $g$ on $A$ which are bounded. Therefore $\mathrm{cl}_{X} A$ has the FCI-property in $X$. Now assume condition (2) and let $x \in X-Z$. Then $\mathrm{cl} x \cap Z=x^{\prime} \cap Z$ is closed in $Z$ and $\operatorname{cl}_{X}\left(x^{\prime} \cap Z\right)=\mathrm{cl} x$ has the FCI-property in $X$. Therefore $x^{\prime} \cap Z$ has the FCI-property in $Z$. By Lemma 8, condition (3) implies any $g: Z \rightarrow R$ extends uniquely to $f: X \rightarrow R$ and so $L(i)$ is an isomorphism.

A subset $Z \subset X$ will be called extension dense or ext-dense in $X$ if it satisfies the conditions of Theorem 9. Thus ext-dense implies fcl-dense implies dense and neither implication is reversible. 
Corollary 10. For each $R$-base $W$ of $M$ in Lat, $\Theta(M, W)$ is ext-dense in $T(M)$.

Proof. In the construction of Theorem 1, $\theta: M \rightarrow L T(M)$ and $\theta_{W}: M \rightarrow$ $L(\Theta(M, W))$ are both lattice isomorphisms and are related by $\theta_{W}=L(i) \theta$. Hence $L(i)$ is an isomorphism. Let $Z=\Theta(M, W)$ and $X=T(M)$. Suppose $x \in X-Z$ has a neighborhood $U$ with $U \cap \operatorname{cl} x \cap Z=\varnothing$. Then $f$ defined by $f(y)=0$ if $y \in \operatorname{cl} x, f(y)=2$ if $y \in X-\operatorname{cl} x$ and $g$ defined by $g(y)=0$ if $y \in \mathrm{cl} x \cap(X-U)$, $g(y)=1$ if $y \in \operatorname{cl} x \cap U$, and $g(y)=2$ if $y \in X-\operatorname{cl} x$ are two different continuous functions on $X$ whose restrictions to $Z$ are the same. This contradicts $L(i)$ being one to one. Hence no such $x$ can exist and $Z$ is fcl-dense in $X$. Then by Theorem $9, \Theta(M, W)$ is ext-dense.

Theorem 11. For $M \in L$ Lat, the collection of all spaces $X$ with $L(X)$ isomorpbic to $M$ consists of all spaces homeomorphic to either $T(M)$, which is an fc space, or an ext-dense subspace of $T(M)$.

Proof. If $L(X) \approx M$, then by Theorem $6, X$ is homeomorphic to $\Theta(M, W)$ which, by Corollary 10, is ext-dense in $T(M)$. Since $M$ is isomorphic to $L T(M)$, $T(M)$ is homeomorphic to $T(L T(M))$. But by $[6, \$ 4], T(L(Y))$ is an fc space for any space $Y$. Note that this theorem determines the inverse image of the epireflection onto the $\mathrm{fc}$ spaces defined in [6].

Proposition 12. A proper ext-dense subspace $Z$ of an fc space $X$ is not an fc space.

Proof. Suppose $x \in X-Z$ where $Z$ is ext-dense in the fc space $X$. Since $\mathrm{cl}_{X} x$ is an irreducible closed $\mathrm{FCI}$ set in $X, \mathrm{cl}_{X} x \cap Z$ is irreducible closed and FCI in $Z$. If $Z$ is an fc space, then there is a $z$ such that $\mathrm{cl}_{Z} z=\mathrm{cl}_{X} x$ $\cap Z$. But $\mathrm{cl}_{Z} z=\mathrm{cl}_{X} z \cap Z$. So by Lemma $7, \mathrm{cl}_{X} Z=\mathrm{cl}_{X} x$ or $z=x$ which is a contradiction.

Proposition 13. If $f: Y \rightarrow Z$ is a bomeomorphism between two ext-dense subsets of an fc space $X$, then $f$ extends uniquely to an ambient homeomorphism b: $X \rightarrow X$.

Proof. Let $x \in X$. Then $\mathrm{cl} x \cap Y$ is an irreducible closed FCI-set of $Y$ hence $f(\mathrm{cl} x \cap Y)$ is an irreducible closed FCI set of $Z$. Therefore cl $f(c l x \cap Y)$ is an irreducible closed FCI set of $X$ and as such is the closure of a point, say $w$. Thus $f(c l x \cap Y)=c l w \cap Z$. Define $b(x)=w$. The same construction using the homeomorphism $f^{-1}$ produces the inverse of $b$, so $b$ is one to one and onto. If $y \in Y, f(\operatorname{cl} y \cap Y)=f\left(\operatorname{cl}_{Y} y\right)=\mathrm{cl}_{Z} f(y)=\mathrm{cl} f(y) \cap Z$ and also $f(\mathrm{cl} y \cap Y)=\mathrm{cl} b(y) \cap Z$. Thus $\mathrm{cl} b(y)=\mathrm{cl} f(y)$ by Lemma 7 , or $b(y)=f(y)$ 
and $b$ extends $f$. To show $b$ is a closed map let $V$ be a closed subset of $X$. Then $V \cap Y$ is closed in $Y$ and $f(V \cap Y)$ is closed in $Z$. Therefore there is a unique closed set of $X, W$, such that $f(V \cap Y)=W \cap Z$. To show $b(V)=W$ let $p \in V$. Then $\mathrm{cl} p \cap Y \subset V \cap Y$, so $(\operatorname{cl} b(p)) \cap Z=f(\mathrm{cl} p \cap Y) \subset f(V \cap Y)=W \cap$ $Z$. Since $Z$ is fcl-dense, $\operatorname{cl} b(p) \subset W$ or $b(p) \in W$ and $b(V) \subset W$. Similarly $b^{-1}(W) \subset V$ or $W \subset b(V)$ and so $b(V)=W$ is closed. Likewise $b^{-1}$ is a closed map and $b$ is a homeomorphism. The extension $b$ is unique because $Y$ is fcldense.

Recall that a pc space [6] is a space where every irreducible closed set is the closure of some point. A $T_{D}$ space [2] is a space where the derived set $x^{\prime}$ is closed for each $x \in X$. A space $X$ has the property that $C(X)$ isomorphic to $C(Y)$ implies $X$ is homeomorphic to $Y$ iff $X$ is both a pc and a $T_{D}$ space (see [4] or [3]). To obtain the corresponding theorem for the lattices $L(X)$, both categories of spaces need to be enlarged. The fc spaces properly contain the pc spaces. A space $X$ will be called a $T_{P}$ space if for each $x \in X$, either $x$ is a $G_{\delta}$ set or $x^{\prime}$ is closed. Thus the $T_{P}$ spaces properly contain the $T_{D}$ spaces.

Lemma 14. $X$ bas no proper ext-dense subspaces iff $X$ is a $T_{P}$ space.

Proof. Suppose $X$ has no proper ext-dense subspaces and let $x \in X$. If $x^{\prime}$ is closed, $X-x$ is not fcl-dense. If $x^{\prime}$ is not closed, $X-x$ is fcl-dense and so there must be a map $g: X-x \rightarrow R$ which is unbounded on $x^{\prime}$. Define $U_{n}=$ $g^{-1}((n, \infty))$. Then $U_{1} \supset U_{2} \supset \cdots$ is a sequence of nonempty open sets, each meeting $x^{\prime}$ and $\cap U_{n}=\varnothing$. Let $V_{n}$ be the unique open set of $X$ with $V_{n} \cap$ $(X-x)=U_{n}$. If $x \notin V_{n}$ then cl $x \subset X-V_{n}$ or cl $x \subset X-U_{n}$ which contradicts $x^{\prime} \cap U_{n} \neq \varnothing^{n}$. Therefore $x \in V_{n}$ or $U_{n} \cup x^{n}=V_{n}$ is open. Thus $V_{1} \supset V_{2} \supset \ldots$ is a sequence of open sets, $\bigcap^{n} V_{i}=x, x$ is a $G_{\delta}$ set, and $X$ is a $T_{P}$ space.

Conversely, suppose $Z$ is an ext-dense subset of the $T_{P}$ space $X$. If $x \in X-Z$, since $Z$ is fcl-dense, $x^{\prime} \cap Z$ has $x$ as a limit point. In particular $x^{\prime}$ is not closed, so open sets $V_{1} \supset V_{2} \supset \cdots$ exist with $\bigcap V_{i}=x$. Define $f$ : $X-x \rightarrow R$ by $f(y)=n$ if $y \in V_{n}-V_{n+1}$. Since $\cap V_{i}=x, f$ is defined for all $y \neq x$. If $m$ is the greatest integer less than or equal $r+1, f^{-1}((-\infty, r])=$ $(X-x)-V_{m}$ and hence $f$ is continuous. Each $V_{n}$ meets $x^{\prime}$ so $f$ is unbounded on $x^{\prime}$. Let $g=f \mid Z$. Then $g$ is bounded above on $y^{\prime} \cap Z$ by $g(y)$. If $g$ is bounded above on $x^{\prime} \cap Z$, by Lemma 8 there is an extension $k: X \rightarrow R$ of $g$. Then $k \mid X-x$ is an extension of $g$ and by uniqueness, $f=k \mid X-x$. However $k$ is bounded above on $x^{\prime}$ by $k(x)$. Therefore $g$ is not bounded above on $x^{\prime} \cap$ $Z$ and $g$ does not extend to all of $X$. This means $Z$ cannot be a proper extdense subset of $X$. 
Theorem 15. $X$ bas the property that $L(X)$ isomorphic to $L(Y)$ implies $X$ is bomeomorpbic to $Y$ iff $X$ is an fc and $T_{P}$ space.

Proof. Suppose $L(X)$ isomorphic to $L(Y)$ implies $X$ is homeomorphic to $Y$. Then since $L(X) \approx L(T L(X)), X$ is homeomorphic to $T L(X)$ which is an fc space by Theorem 11. If $X$ is not a $T_{P}$ space, by Lemma 14 it has a proper ext-dense subset $Z$. By Theorem $9, L(X) \approx L(Z)$, yet $X$ is not homeomorphic to $Z$ since by Proposition 12, $Z$ is not an fc space. Therefore $X$ is both an $\mathrm{fc}_{\mathrm{c}}$ and $T_{P}$ space. Conversely let $X$ be an fc and $T_{P}$ space. By Theorem 6 and Corollary 10 , if $L(X) \approx L(Y) \approx M$, both $X$ and $Y$ are homeomorphic to ext-dense subsets of $T(M)$. But $T(M)=X$ since both are fc spaces and $Y=X$ since $X$ has no proper ext-dense subsets.

From the proof of Lemma 14 it is clear that if $Z$ is ext-dense in $X$ and $x$ is either a $G_{\delta}$ set or has $x^{0}$ closed, then $x \in Z$. Since $\Theta(M, W)$ is ext-dense in $T(M)$ this means that if $P(A)$ is a bounded class of closed prime ideals in $M$ and either $P(A)^{\prime}$ is closed in $T(M)$ or $P(A)$ is a $G_{\delta}$ set, then $P(A) \epsilon$ $\Theta(M, W)$, that is $P(A)$ must be in the basis $W$. Hence the intersection of all $R$ bases of $M$ contains all those bounded classes which are $G_{\delta}$ in the hull-kernel topology or have hull-kernel closed derived sets. It follows that if the collection of all such bounded classes forms an $R$-basis, it must be a minimum bas is $W_{m}$. Then $\Theta\left(M, W_{m}\right)$ is a $T_{P}$ space which is homeomorphic to an ext-dense subset of any space $Y$ with $L(Y) \approx M$. Conversely, if $M$ has a representation as $L(Z)$ where $Z$ is a $T_{P}$ space, then such a minimum $R$-basis for $M$ exists. $Z$, if it exists, can be considered as a minimum space in the collection of all spaces $X$ with $L(X) \approx M$ just as the fc space $T(M)$ can be considered as the maximum space. These remarks prove our last theorem. The corresponding result for $\mathrm{fc}$ spaces is already known [6, Theorem 4.5].

Theorem 16. Let $X$ and $Y$ be two $T_{P}$ spaces. Then $L(X) \approx L(Y)$ iff $X$ is bomeomorphic to $Y$.

\section{REFERENCES}

1. F. W. Anderson and R. L. Blair, Characterizations of the algebra of all realvalued continuous functions on a completely regular space, Illinois J. Math. 3 (1959), 121-133. MR $20 \# 7214$.

2. C. E. Aull and W. J. Thron, Separation axioms between $T_{0}$ and $T_{1}$, Nederl. Akad. Wetensch. Proc. Ser. A 65 = Indag. Math. 24 (1962), 26-37. MR 25 \#1529.

3. T. Blanksma, Lattice characterizations of topologies and compactifications, Doctoral Dissertation, University of Utrecht, Groningen, 1968. MR 37 \#5851.

4. D. Drake and W. J. Thron, $O n$ the representations of an abstract lattice as the family of closed sets of a topological space, Trans. Amer. Math. Soc. 120 (1965), 57-71. MR $32 \# 6390$. 
5. L. D. Nel, Lattices of lower semi-continuous functions and associated topological spaces, Pacific J. Math. 40 (1972), 667-673.

6. L. D. Nel and R. G. Wilson, Epi reflections in the category of $T_{0}$-spaces, Fund. Math. 75 (1972), 69-74.

7. L. Skula, $O_{n}$ a reflective subcategory of the category of all topological spaces, Trans. Amer. Math. Soc. 142 (1969), 37-41. MR 40 \#1969. 68508

DEPARTMENT OF MATHEMATICS, UNIVERSITY OF NEBRASKA, LINCOLN, NEBRASKA 